\title{
Modelo de manejo de datos, con el uso de inteligencia artificial, para un sistema de información geográfica en el sector energético
}

\section{(Data model management, with the use of artificial intelligence, for a geographic information system in the energetic sector)}

\author{
Nayi Sánchez Fleitas ${ }^{1}$, Raúl Comas Rodríguez², María Matilde García Lorenzo ${ }^{3}$, Amanda Riverol
} Quesada $^{4}$

\begin{abstract}
Resumen:
Para el sector eléctrico se desarrolla un Sistema de Información Geográfica denominado SIGOBE versión 3.0. Las bases de datos que tributan información alfanumérica son el Sistema Integral de Gestión de la ECIE (SIGECIE) y el Sistema Integral de Gestión de Redes (SIGERE). Estudios realizados determinan la necesidad de un modelo para el manejo de datos, que contribuya al desarrollo del SIG, sobre un esquema conceptual del dominio capaz de dar respuestas a las diferentes peticiones del usuario, por medio de consultas automáticas, como soporte a la toma de decisiones. Para dotar al SIG de una base conceptual se desarrolla una ontología, expresada mediante lógicas descriptivas, para generar los rasgos de un Razonamiento Basado en Casos que permite la automatización de las consultas. La calidad final del SIG se verifica de acuerdo con los estándares de calidad de la norma ISO-9126:2002. El modelo propuesto y sus funcionalidades contribuye a: facilitar la toma de decisiones a diferentes niveles, realizar análisis de riesgos al tener los defectos de las instalaciones eléctricas, disminuir el tiempo de avería a las áreas claves del país, organizar el recorrido de los carros más eficientemente y localizar las fallas eléctricas con mayor precisión.
\end{abstract}

Palabras clave: Sistema de Información Geográfica; ontologías; razonamiento basado en casos; sector energético

\begin{abstract}
:
A Geographic Information System (GIS), named SIGOBE v 3.0, for the electric sector is development. The Integral Management System of the ECIE (SIGECIE) and the Integrated Network Management System (SIGERE) databases are taxed alfanumeric information. Studies determined the need for a model for data management, contributing to the GIS development, on a conceptual schema domain capable of responding to different user requests, through automatic query as support decision making. To provide the GIS with a conceptual basis an ontology is determined, which will be expressed by logical descriptive, to generate the traits of a case-based reasoning that allows automation of consultations. The final quality of GIS was verified according to the quality standards of the ISO-9126:2002 standard. The proposed model and its functionality contributes to: facilitate decision-making at different levels, perform risk analysis to have the defects of electrical installations, reduce the time of failure to the key areas of the country, organize the travel of trucks more efficiently and locate electrical faults more accurately.
\end{abstract}

Keywords: Geographic Information System; ontologies; case-based reasoning; energy sector

\footnotetext{
${ }^{1}$ UEB Aplicaciones de las Tecnologías Informáticas, Sancti Spíritus - Cuba (nayi@atiss.une.cu)

2 Universidad Técnica de Ambato, Ambato-Ecuador (ra.comas@uta.edu.ec)

3 Universidad Central Marta Abreu de Las Villas, Santa Clara - Cuba (mmgarcia@uclv.edu.cu)

${ }^{4}$ Universidad de Cienfuegos, Cienfuegos - Cuba (ariveroll@ucf.edu.ec)
} 


\section{Introducción}

La energía eléctrica es determinante para el desarrollo de un país y requiere de una compleja infraestructura para su uso y control (Ibarra Ruiz, 2013). El proceso energético pone en funcionamiento un amplio complejo industrial y equipos que se emplean en todas las ramas de la economía y la vida social. El sector energético, por las características de la sociedad actual, forma parte importante en elevar la calidad de vida humana (González, Pérez, Vásquez Stanescu, \& Araujo, 2014).

Las empresas eléctricas se sostienen en tres pilares claves: la generación, trasmisión y distribución de la energía (Short, 2004). Los Sistemas de Gestión Empresarial se centran en la informatización del control de los procesos eléctricos como parte del proceso de automatización (Fernández 2011). El el Sistema Integral de Gestión de Redes (SIGERE) y el Sistema Integral de Gestión de la ECIE (SIGECIE) son Sistemas de Información Gerencial, que recogen datos técnicos, económicos y de gestión para convertirlos en información. Los datos recopilados facilitan y mejoran la eficiencia en la operación, explotación, análisis, planificación y gestión de las redes eléctricas de distribución y transmisión. Como parte de los proyectos se desarrolla un Sistema de Información Geográfica (SIG) llamado SIGOBE que se encuentra en su versión 3.0.Un SIG es utilizado para la visualización y el análisis de la información geoespacial. En su origen se desarrollaron para crear mapas temáticos que permiten la captación, almacenamiento y el análisis de datos; con la posibilidad de relacionar información detallada en un contexto espacial que permita la mejora en el proceso de toma de decisiones (Garea Llano, 2007).

EI SIGOBE es la interfaz gráfica del SIGECIE y el SIGERE que brinda información geográfica y eléctrica, permitiendo la reducción de los costos operativos y la mejora de la calidad del servicio al cliente. La primera versión del SIG (SIGOBE 1.0) se pone a disposición de los usuarios en el 2001 con 220 opciones de búsqueda. EI SIGOBE manipula la información alfanumérica y cartográfica que tributan estos sistemas de gestión enlazándolas a la cartografía por medio de los códigos de los elementos.

Para el posterior desarrollo y actualización del sistema, en su versión 3.0 se realiza un estudio de los SIG desarrollados en el campo de la energía eléctrica y con un grupo de expertos, especialistas en el tema, se detectan como principales limitaciones: rudimentarios métodos de elaboración de consultas, sistema limitado de consultas fijas, necesidad de una correspondencia semántica entre las bases de datos y los datos geográficos, falta de una base conceptual, y existencia de errores topológicos y de precisión en la base cartográfica empleada.

El análisis realizado permite establecer la necesidad de un modelo para el manejo de datos, que contribuya al desarrollo del SIG, sobre un esquema conceptual del dominio capaz de dar respuestas a las diferentes peticiones del usuario, por medio de consultas automáticas, como soporte a la toma de decisiones. I objetivo general de esta investigación es establecer un modelo 
para el manejo de datos de diferentes dominios, con el uso de la inteligencia artificial, como soporte a la toma de decisiones para los procesos de transmisión y distribución de la energía eléctrica.

\section{Metodología}

Los SIG son una representación de la realidad digitalizada. El almacenamiento de sus datos se puede realizar en forma: de vector o imágenes raster. Los datos del SIGOBE se almacenan de forma vectorial a partir de líneas, puntos y polígonos. Cada tema diferente se guarda en una capa separada y todos los elementos areales se representan por polígonos cerrados.

Las pruebas pilotos realizadas permiten definir el uso de las escalas siguientes: 1:2000 para el área urbana, 1:5000 para asentamientos rurales y 1:100 000 para las provincias con un margen de error de 1 metro, 2,5 metros y 50 metros respectivamente. La base cartográfica eléctrica se encuentra georreferenciada solo a partir de los postes, que son los puntos de apoyo del sistema eléctrico, y el resto de los componentes se encuentran en las bases de dato, que permiten una actualización automática de toda la información necesaria. Un equipo de desarrollo se encarga de la realización de limpieza topológica de cada una de las capas que componen la cartografía y de integrar las escalas.

Los SIG se desarrollan generalmente enfocados en un contexto particular y con restricciones dentro de un dominio específico. En ocasiones diversas entidades tienen deficiencias en la comunicación por falta de un entendimiento cooperado al definir objetos iguales de formas diferentes, a partir de puntos de vistas y supuestos en un dominio de estudio determinado (Durango Vanegas, 2015; Larin Fonseca \& Garea Llano, 2009).

En este sentido, para la solución de problemas derivados de la heterogeneidad e interoperabilidad de los datos, una de las herramientas de mayor uso son las ontologías (Machado García, González Ruiz, \& Balmaseda Espinosa, 2014).

La palabra ontología se establece de un vocablo griego que significa "estudio del ser" (De La Peña, 2013). Los fundamentos del vocablo provienen de los filósofos Sócrates y Aristóteles. Sócrates introduce nociones sobre ideas abstractas, la jerarquía de estas ideas y relaciones clase - instancia; Aristóteles le integra lógicas asociativas (Vera \& Garea Llano, 2009). La definición del término ontología ha evolucionado y es considerada como "una especificación formal y explícita de una conceptualización" (Gruber, 1993).

Dentro de la informática el término ontología hace referencia a la formulación de un exhaustivo y riguroso esquema conceptual dentro de un dominio dado, con la finalidad de facilitar la comunicación y el intercambio de información entre diferentes sistemas. Esta la diferencia con el 
significado etimológico de la palabra ontología, tomando su nombre por analogía (Aguilar \& Moreno, 2014; Vera \& Garea Llano, 2009).

Una ontología define los términos usados sobre: un área del conocimiento, las relaciones básicas entre ellos, las reglas para combinarlos y sus extensiones. Cuando se representan los términos mediante la lógica descriptiva se pueden utilizar en procesos de: clasificación, reconocimiento de instancias, relaciones de subfunción y orden parcial entre los conceptos. Lo anterior permite el desarrollo sobre una profunda base conceptual para la elaboración de consultas automáticas (Rivero Hernández, Vila Labrada, \& Rivero Hernández, 2014).

Para el desarrollo del modelo propuesto en la investigación, se determina una base conceptual, con el desarrollo de una ontología ligera (Figura 1). En la conceptualización se formulan los conceptos, con su taxonomía y relaciones (propiedades de objetos). Los demás componentes en el modelo de la ontología propuestas (las propiedades en los datos, las instancias y los axiomas) están en la base de datos que alimenta el sistema. Para el desarrollo del modelo se utiliza como metodología de desarrollo Methontology (Fernández \& Gómez Pérez, 2002) y como editor la herramienta Protégé ${ }^{5}$.

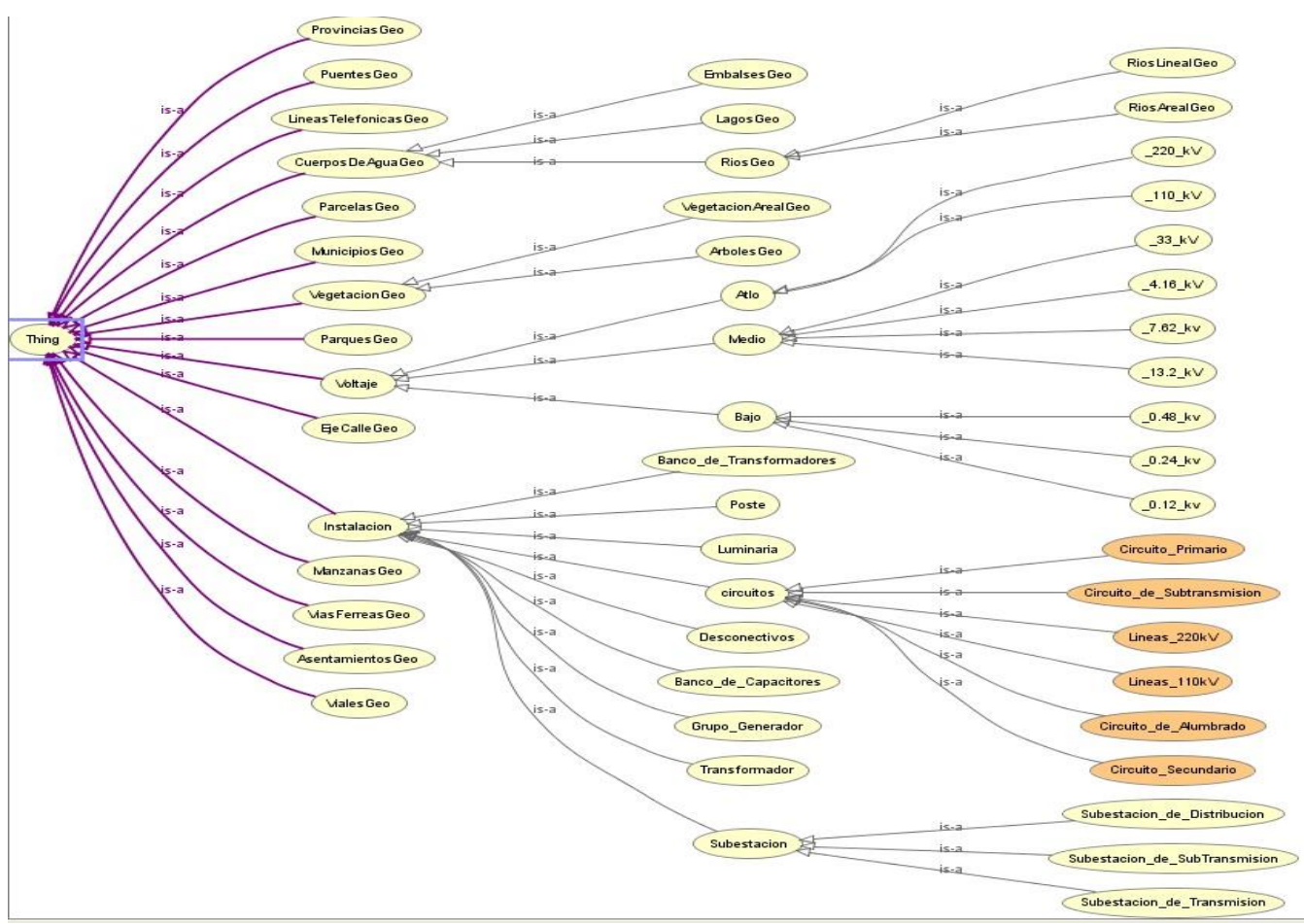

Figura 1. Esquema de representación de los conceptos y taxonomía de la ontología para el SIG

El desarrollo de las consultas automáticas se realiza a partir de las 220 consultas definidas y el principio que: "la solución de un nuevo problema se realiza a partir de las soluciones conocidas

\footnotetext{
${ }^{5}$ Protégé es una herramienta Open Source concebida como editor de ontologías y un framework para la construcción de sistemas inteligentes realizado por la Universidad de Stanford y disponible en http://protege.stanford.edu/
} 
para un conjunto de problemas previamente resueltos (o no resueltos) del dominio de aplicación" (Febles Díaz, Febles Rodríguez, Estrada Sentí, \& Díaz Márquez, 2012, p. 300).

Adicionalmente, el Razonamiento Basado en Casos (RBC) es un método para resolver problemas no estructurados, donde se realiza el razonamiento desde una memoria asociativa a partir de un algoritmo capaz de formalizar medidas de semejanza para dos objetos. La base del comportamiento inteligente de un sistema radica en el paradigma de recordar situaciones similares existentes en el pasado. El RBC es una técnica, en la cual la memoria se sitúa como fundamento de la Inteligencia Artificial y concretamente de los sistemas basados en el conocimiento (Febles Díaz et al., 2012).

Los análisis realizados permiten proponer para el modelo un sistema de RBC que cuenta con tres componentes principales: una base de casos (BC), un motor de inferencia y una interfaz de usuario.

\subsection{Base de casos}

La BC se formula con la descripción de consultas estáticas realizadas previamente en forma de casos. Cada consulta se compone por once rasgos fundamentales derivados de la ingeniería del conocimiento realizada: ocho rasgos predictores y tres objetivos. La Figura 2 muestra la estructura de la base de casos y la Tabla 1 las características básicas de cada rasgo predictor.

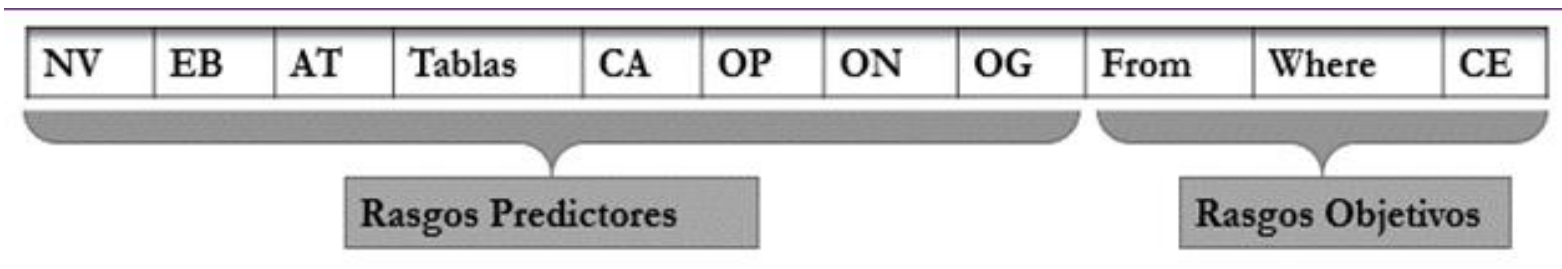

Figura 2. Estructura de un Caso.

Tabla 1. Características básicas de los rasgos predictores, valores y tipo.

\begin{tabular}{|c|c|c|}
\hline Rasgo & Posibles valores & Tipo \\
\hline NV & Secundario, Primario, Subtransmisión, Transmisión & $\begin{array}{l}\text { simbólico y } \\
\text { univaluado }\end{array}$ \\
\hline EB & $\begin{array}{l}\text { Postes, Bancos de transformadores, Bancos de capacitores, Grupos } \\
\text { generadores, Desconectivos, Estructuras, Luminarias, Circuito } \\
\text { Transmisión, Circuito Subtransmisión, Circuito Primarios, Circuito } \\
\text { Secundarios, Circuito Alumbrado, Subestación de Distribución, } \\
\text { Subestación de Transmisión }\end{array}$ & $\begin{array}{l}\text { simbólico y } \\
\text { univaluado }\end{array}$ \\
\hline AT & Atributos que debe devolver la consulta $X=\{x 1, x 2, \ldots, x k\}$ & Conjunto \\
\hline Tablas & $\begin{array}{l}\text { Tablas involucradas en la consulta (Accesorios, Acciones, Acometidas, } \\
\text { Afectaciones_Linea, ActualAlimemtacionPrimaria, }\end{array}$ & Conjunto \\
\hline $\mathrm{CA}$ & Elemento a comparar (Atributo sobre el cual se realiza la comparación) & $\begin{array}{l}\text { simbólico y } \\
\text { univaluado }\end{array}$ \\
\hline OP & Operador $(U, \cap, \leq \geq=$, like, etc $)$ & $\begin{array}{l}\text { simbólico y } \\
\text { univaluado }\end{array}$ \\
\hline ON & Ontología (lógica descriptiva)( $\mathrm{T} \cap \mathrm{TP} \cap \mathrm{TM}$ onofásicos $\neg S$ Secundaria) & Ontología \\
\hline $\mathrm{OG}$ & Restricción espacial (lógica descriptiva) & Ontología \\
\hline
\end{tabular}


Los rasgos objetivos son de tipo cadena y su objetivo es:

- From: Devuelve el From de la consulta al SIGERE.

- Where: Devuelve el Where de la consulta al SIGERE.

- ConOE : Devuelve la consulta al SIG

Para organizar la base de casos se utiliza una estructura jerárquica que favorece al sistema, el proceso de acceso y recuperación de los ejemplos más similares a la consulta en tiempo real.

\subsection{Motor de inferencia}

El motor de inferencia es la máquina de razonamiento del sistema, el cual compara el problema insertado con los que están almacenados en la base de casos. Como resultado infiere una respuesta con el mayor grado de semejanza a la que se busca, adaptando los casos más similares recuperados. (Cordero Morales, Ruiz Constanten, \& Torres Rubio, 2013)

El razonador sigue el ciclo de las 4R (Figura 3) que incluye las etapas de recuperar problemas 0 casos similares y enviarlos al módulo adaptador, con el fin de dar la solución de la forma más óptima al problema a resolver y reutilizando las soluciones propuestas en los casos recuperados. Una vez hallada la solución, de ser necesario se revisa y se retiene junto con la descripción del problema en la BC. La solución definida constituye un nuevo caso que se inserta en la BC.

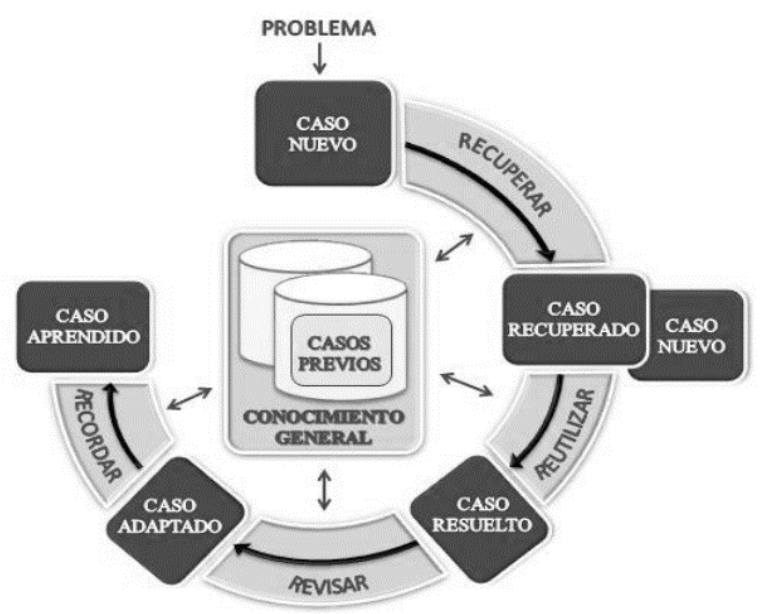

Figura 3: Ciclo Razonador Basado en Casos. (Cordero Morales et al., 2013)

\subsubsection{Módulo recuperador}

El módulo recuperador se encarga de extraer de la BC el caso o los casos más parecidos a la situación actual con el uso de una medida de similitud efectiva. El concepto de similitud es la generalización de la similitud entre dos triángulos en el plano y entre dos matrices en la matemática. La similitud entre un caso y un problema debe corresponderse con la utilidad o reusabilidad del caso para establecer la solución del problema, es decir, es deseable recuperar 
casos previos que sean fácilmente adaptables al nuevo problema y/o tengan soluciones similares a este (Morell Pérez, 2005).

En correspondencia con el tipo de información que se represente existen distintas definiciones de similitud, disimilitud o distancias (Bonillo, 2003). Si se tiene en cuenta que un caso viene descrito por un conjunto de atributos, la aproximación más básica para el cálculo de la similitud consiste en contabilizar los valores iguales en los atributos comunes de los casos a comparar.

El valor asignado a un atributo de evaluación para indicar su importancia relativa con respecto a los otros atributos es el peso. El peso de un atributo debe seleccionarse por criterio de expertos, incrementando o decreciendo el rango de los atributos. En el caso de $n$ atributos, el conjunto de pesos es definido por la ecuación 1 :

$w=\left(w_{1}, w_{2}, \ldots w_{i}, \ldots, w_{n}\right) w_{i} \geq 0$ y $\sum w_{j}=1$

El éxito en la estimación de la similitud determina la eficacia del sistema de RBC; varios autores coinciden en señalar que es el punto más complejo al implementar estos sistemas (Atkeson, Moore, \& Schaal, 1997; Bergmann \& Stahl, 1998).

El recuperador del sistema obtiene los $\mathrm{k}$ casos más cercanos a la consulta solicitada por el usuario, tomando para el cálculo de la distancia la ecuación 2, que determina la similitud global. Como resultado de la evaluación del sistema, el k seleccionado por defecto es 3 . El conjunto de casos obtenidos constituye la entrada del módulo adaptador. La similitud global es el resultado de la suma ponderada de las distancias entre el valor de cada rasgo en un caso y el valor que toma el mismo en el caso problema. Las distancias son ponderadas según el criterio de expertos, con un peso $\mathrm{w}_{\mathrm{i}}$, para hacer que el caso problema se acerque más a un caso en particular; $\mathrm{w}_{\mathrm{i}}$ es definido debido que los rasgos no tienen la misma importancia, es decir, mientras mayor sea $w_{i}$ mayor será la importancia del rasgo.

$\operatorname{SimGlobal}(X, Y)=\sum_{i=0}^{m} w_{i} * \frac{d_{i}\left(x_{j}, y_{j}\right)}{n}$

Donde $\sum w_{i}=1$

La distancia local $\mathrm{d}_{\mathrm{i}}\left(\mathrm{x}_{\mathrm{i}}, \mathrm{y}_{\mathrm{i}}\right)$ está determinada por el tipo de datos de $\mathrm{x}_{\mathrm{i}}, \mathrm{y}_{\mathrm{i}}$. En el caso expuesto se cuenta con tres tipos de datos que utilizan diferentes medidas de distancia.

Los rasgos NV, EB, AC y OP son de tipo simbólico univaluado, la distancia utilizada es de tipo booleana. El cálculo de la distancia local se determina a partir de la ecuación 3. Se puede observar que toma valor 1 si el valor $x_{i}$ es distinto al de $y_{i} 0$ valor 0 en caso contrario (Althoff, 1995). 
$d_{i}\left(x_{j}, y_{j}\right)=\left\{\begin{array}{l}0 \text { Si } x_{j} \neq y_{j} \\ 1 \text { Si } x_{j}=y_{j}\end{array}\right.$

Los rasgos AT y Tablas son de tipo conjunto. González Castellanos (2010) analiza un grupo de propuestas para este tipo de distancia que se encuentran en la literatura. Para seleccionar el tipo de distancia se realizan pruebas al sistema y se determina el uso de la distancia Jaccard, basada en las operaciones de conjunto: unión e intercepción (Ecuación 4).

$d_{i}\left(x_{j}, y_{j}\right)=\frac{\left|x_{j} \cap y_{j}\right|}{\left|x_{j} \cup y_{j}\right|}$

Los rasgos OG y OE representan las ontologías general y espacial, respectivamente. Al ser tratados como cadenas cada uno de sus componentes son subcadenas, prestando mayor interés al orden que aparecen estas subcadenas sobre la cantidad de cadenas similares. Para su implantación se analizan los tipos de distancias: Brecha Afín (Gotoh, 1982), Edición (Ramírez Bustamante \& López Díaz, 2006), Smith-Waterman (Smith \& Waterman, 1981), Jaro (Jaro, 1976) y JaroWinkler (Winkler, 2000).

Para la selección, en este caso, se realiza una validación cruzada dejando uno fuera (Leave One Out Crossvalidation), en la cual se separan los datos. Para cada iteración se tiene un caso de prueba y el resto de entrenamiento. Los casos donde se utilizan todos los rasgos, con mayor importancia al rasgo ontología y con adaptación inicial, obtiene los mejores resultados. Dentro de este caso de prueba destaca la medida de similaridad de Jaro-Winkler con un $97 \%$ de efectividad.

En la distancia Jaro-Winkler (ecuación 5): l es la longitud de prefijo común de las cadenas hasta un máximo de cuatro caracteres; p es un factor de escala constante que no debe ser mayor que 0.25 ( 0.1 es el valor estándar que se utiliza), $b_{t}$ es el umbral que se define (se recomienda $0.7), d_{j a r o}$ es la distancia de Jaro entre las cadenas $x_{j}, y_{j}$, que define como operación de edición la transposición de dos caracteres. Estos caracteres no tienen que estar adyacentes, pueden estar a una distancia determinada en función de la longitud de ambas cadenas (ecuación 6) (Winkler, 2000).

$d_{i}\left(x_{j}, y_{j}\right)=\left\{\begin{array}{c}d_{\text {jaro }} \text { si } d_{\text {jaro }}<b_{t} \\ d_{\text {jaro }}+\left(l * p *\left(1-\left|y_{j}\right|\right)\right) \text { e.o.c }\end{array}\right.$

$d_{\text {jaro }}=\frac{1}{3}\left(\frac{c}{\left|x_{j}\right|}+\frac{c}{\left|y_{j}\right|}+\frac{c-t / 2}{c}\right)$

\subsubsection{Módulo adaptador}


El módulo adaptador sigue una analogía transformacional (Figura 4) que parte de transformar la solución de un problema previo en la solución del nuevo problema a resolver. Se considera que existe un T-espacio en el cual la solución conocida puede ser transformada, usando T-operadores, hasta convertirla en la solución de un nuevo problema.

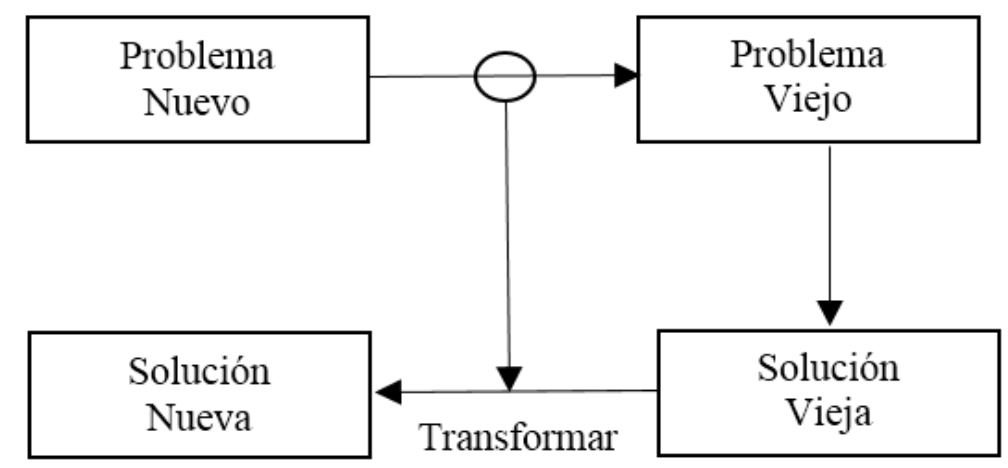

Figura 4: Analogía transformacional

\section{Resultados}

Para la implementación del RBC se desarrolla dentro del SIGOBE un módulo que recibe el nombre de SICUNE (Sistema Inteligente de Consulta) con el uso de analogía transformacional sobre consultas previamente hechas, recuperadas por un razonador basado en casos, para dar respuesta a interrogantes del usuario. Durante este proceso el sistema utiliza las facilidades del API JxI de Java ${ }^{6}$ para manipular la base de casos desde un sistema desktop preparado para el trabajo en red restringido a un segmento de usuarios con acceso a la informaciòn.

La aplicación se estructura en paquetes como mecanismo utilizado para agrupar elementos que facilitan su organización (Figura 5). Los paquetes Set, String y Position almacenan todos los elementos relacionados del trabajo con conjuntos, cadenas y similitud por posición respectivamente. Los paquetes Structure y Useful almacenan todos los elementos relacionados con el acceso y manipulación de la base de casos respectivamente. Todos los paquetes tributan a un paquete RBC Visual que almacena los elementos encargados de establecer el vínculo entre la interfaz y la aplicación.

La interfaz de usuario propuesta permite la comunicación entre el sistema y el usuario, con la posibilidad de interactuar con la base de casos, plantear nuevos problemas y consultar los resultados inferidos. El sistema cuenta con 6 funcionalidades:

- Configuración de medidas de distancia para los rasgos

- Asignación de peso a los rasgos

- Consulta al sistema

${ }^{6}$ Código abierto de Java API que permite leer, escribir y actualizar las hojas de cálculo de Excel 
- Observación de la Base de Casos en su totalidad o la sub-base en la cual se está recuperando

- Cargar un conjunto de casos a consultar

- Almacenar la respuesta al conjunto de datos de los casos cargados

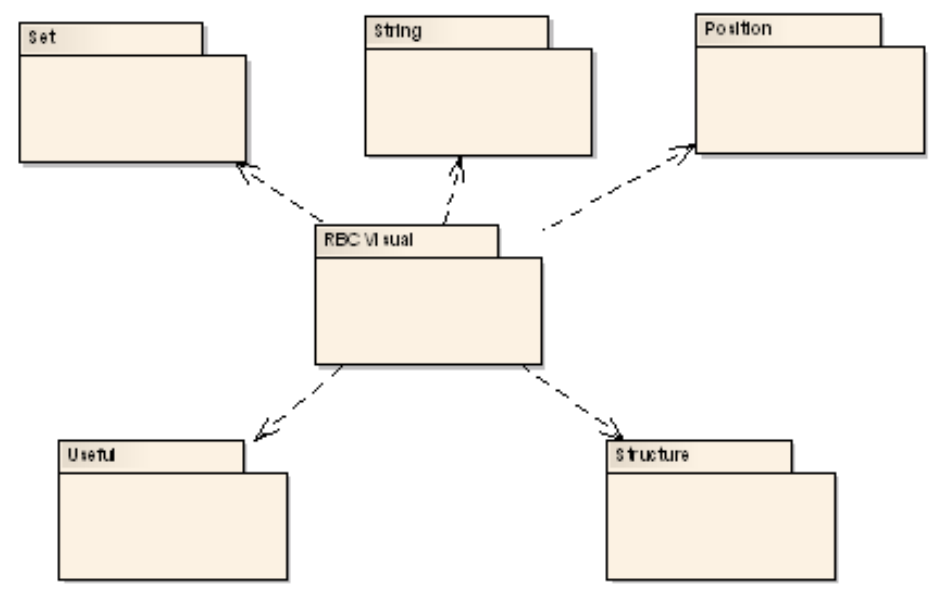

Figura 5: Paquetes del SICUNE

La versión del SIGOBE que incluye el SICUNE (Figura 6), se implanta en una empresa eléctrica para realizar las pruebas pertinentes y finalmente desplegarlos por todo el país.

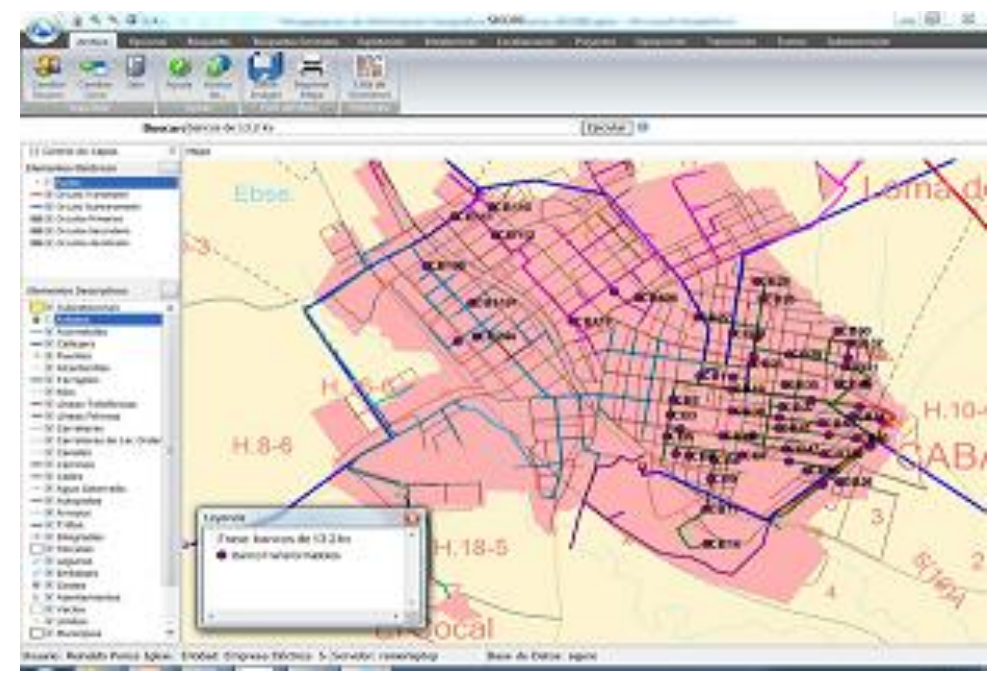

Figura 6. Funcionalidad implementada.

Para la evaluación del sistema se utiliza un modelo de evaluación propuesto por Guzmán (2009), basado en la norma internacional ISO-9126:2002 que evalúa la calidad de sistemas informáticos con el uso de métricas. La norma ISO-9126:2002 define seis características básicas para evaluar calidad tanto interna como externa de un sistema informático: funcionalidad, confiabilidad, usabilidad, eficiencia, capacidad de mantenimiento y portabilidad. Adicionalmente propone cuatro características para evaluar la calidad en su uso: eficacia, productividad, seguridad y satisfacción. 
La aplicación de la evaluación en la investigación permite definir las fortalezas y debilidades que el modelo ofrece y si satisface las expectativas esperadas. Para la evaluación se utilizan los atributos del modelo: Confiabilidad (At2), Usabilidad (At3), Eficiencia (At4), Capacidad de mantenimiento (At5), Portabilidad (At6). Como complemento del proceso de evaluación para el análisis de su uso se tiene en cuenta adicionalmente: Eficacia (Au1), Productividad (Au2), Satisfacción (Au3), Seguridad (Au4).

En la Tabla 2 se muestra el resultado de la valoración de 7 expertos consultados, que otorgaron pesos a cada una de las características de calidad; cada indicador se refleja en base a $10 \%$.

Tabla 2: Evaluación de los atributos de Calidad de Software

\begin{tabular}{|l|c|c|c|c|c|c|c|}
\hline \multirow{2}{*}{} & \multicolumn{6}{|c|}{ Atributos de calidad externa e } & Totales \\
& interna (At) & \\
\cline { 2 - 7 } & At1 & At2 & At3 & At4 & At5 & At6 & \\
\hline Puntos & 10 & 10 & 10 & 10 & 10 & 8 & 58 \\
\hline & \multicolumn{6}{|c|}{ Atributos de calidad en uso (Au) } & \\
\cline { 2 - 6 } & Au1 & Au2 & Au3 & Au4 & \\
\hline Puntos & 9 & 10 & 10 & 9.5 & 38.5 \\
\hline \multicolumn{7}{|c|}{ Puntaje total } & 96.5 \\
\hline
\end{tabular}

En la investigación los atributos evaluados sobre calidad externa e interna obtienen un valor de $10 \%$ en sus parámetros, con excepción de la portabilidad que alcanza un $8 \%$. Los atributos que evalúan la calidad de uso: la productividad y la satisfacción obtienen un 10\%, eficacia un $9 \%$ y seguridad un $9,5 \%$.

El puntaje total en el proceso de evaluación obtiene un 96,5\% de las funcionalidades que se incorporan al SIGOBE a través del SICUNE. El análisis anterior permite considerar apto el producto y establece como debilidad principal la portabilidad, esta valoración se define porque la base de datos de los elementos geográficos no está en un servidor.

La implementación del modelo propuesto permite reducir los gastos de combustible y las horas hombre de trabajo al tener una representación de las redes y la posibilidad de realizar estudios y optimizarlas sin realizar trabajos de campos. Su empleo contribuye a: facilitar la toma de decisiones a diferentes niveles, realizar análisis de riesgos al tener los defectos de las instalaciones eléctricas, disminuir el tiempo de avería a las áreas claves del país, organizar el recorrido de los carros más eficientemente y localizar las fallas eléctricas con mayor precisión

\section{Discusión}

En el ámbito de la información espacial, los esfuerzos para gestionar el conocimiento se centran principalmente en el uso de ontologías y en la inclusión de elementos semánticos en datos geográficos (Oliva Santos, Garea Llano, \& Macia Perez, 2009). 
En el 2009 se presenta Sistema de Información Geográfica Gobernados por Ontología Integrada (SIGGOI) en los que solo existe una componente, la ontología. Ellos incluyen una capa que engloba la información además de enlazar semánticamente y de forma integradora las restantes componentes, también es capaz de contener a sus correspondientes metadatos (Larin Fonseca \& Garea Llano, 2009).

En el 2013 se publica un enfoque de integración semántica de datos geoespaciales a bajo nivel de abstracción. La investigación se basa en el uso de la Ontología de Representación de Datos (ORD) para la representación explícita de la naturaleza semántica de los geodatos, las relaciones entre ellos y posteriormente su vinculación con las diferentes Ontologías de Niveles Superiores (ONS) en dependencia del enfoque de integración semántica a alto nivel utilizado (Larin Fonseca \& Garea Llano, 2013).

En el 2014 se propone la Metaontología geoespacial, 5-tupla (Tolaba, Caliusco, \& Galli, 2014):

Metaontología $=\{C, R, A, X, I\}$

Donde:

C: conjunto de conceptos

$\mathrm{R}$ : conjunto de relaciones entre los conceptos

A: conjunto de atributos de los objetos identificados en el dominio,

$\mathrm{X}$ : conjunto de axiomas y reglas de los conceptos, relaciones y atributos

I: conjunto de instancias

El empleo de los enfoques anteriores en los SIG aporta una conceptualización del sistema y ayudan a representar el conocimiento que existe en las bases de datos de forma más clara y coherente; pero tiene como limitación que el SIG queda restringido a un número de consultas prestablecidas ya sea por el sistema o las relaciones de los conceptos.

Dentro del dominio de investigación, en el campo de la energía eléctrica, la información solicitada por los usuarios es muy variada; de allí la necesidad de generación de consultas automáticas que supere el modelo tradicional y use herramientas de inteligencia artificial como el RBC. En la Base de Casos propuesta se le incorporan cotidianamente nuevos casos que permiten que el sistema se fortalezca, aprenda por sí solo e incorpore nuevo conocimiento. La ontología propuesta, con un lenguaje cercano al natural, resulta cómodo y ágil para los usuarios incrementando el espectro de peticiones en el dominio. 


\section{Conclusiones y Recomendaciones}

La informatización del proceso de transmisión y distribución de la energía eléctrica constituye una prioridad de estos sectores por la importancia en el desarrollo económico y social de las naciones.

La realización de las solicitudes geográficas se gestiona con mayor facilidad y comodidad a partir del procesamiento en lenguaje natural ejecutado por el SIGOBE.

Se diseñó un sistema basado en casos tipo solucionador de problemas, utilizando como base de casos inicial las 265 consultas estáticas registradas en el SIG; las consultas se describen por ocho rasgos predictores de tipos de datos: nominal, conjunto, ontologías y tres rasgos objetivos.

Se implementa un sistema inteligente de consultas en tiempo real para la UNE (SICUNE), lográndose la generación de consultas automáticas, que le permiten al sistema dar respuesta a cualquier tipo de consultas en tiempo real.

La validación del sistema se realizó con casos de prueba obteniéndose un $97 \%$ de exactitud en las respuestas esperadas correspondiéndose con todas las reglas de adaptación y revisión propuesta. El 3\% de error viene dado porque aún es insuficiente los casos almacenados, lo cual se encuentra en vías de solución.

La evaluación de la propuesta desarrollada, mediante la metodología basada en la norma ISO/IEC 9126:2002, y el análisis realizado por un equipo de expertos, corroboró la capacidad del SICUNE, mediante consultas automáticas, para solucionar las peticiones realizadas al SIG por especialistas.

\section{Bibliografía}

Aguilar, J., \& Moreno, K. (2014). Metodología para el Desarrollo de Aplicaciones Basado en el Paradigma ODA. Universidad de Los Andes Mérida, Venezuela.

Althoff, K. D. (1995). A review of industrial case-based reasoning tools: A1 Intelligence.

Atkeson, C. G., Moore, A. W., \& Schaal, S. (1997). Locally Weighted Learning. Artif. Intell. Rev., 11(1-5), 11-73. doi: 10.1023/a:1006559212014

Bergmann, R., \& Stahl, A. (1998). Similarity measures for object-oriented case representations. In B. Smyth \& P. Cunningham (Eds.), Advances in Case-Based Reasoning: 4th European Workshop, EWCBR-98 Dublin, Ireland, September 23-25 (pp. 25-36). Berlin, Heidelberg: Springer Berlin Heidelberg.

Bonillo, M. L. (2003). Razonamiento Basado en Casos aplicado a Problemas de Clasificación. (Doctoral), Universidad de Granada. 
Cordero Morales, D., Ruiz Constanten, Y., \& Torres Rubio, Y. (2013). Sistema de Razonamiento Basado en Casos para la identificación de riesgos de software. Revista Cubana de Ciencias Informáticas, 7(2), 222-239.

De La Peña, J. L. C. (2013). Evolución histórica del Pensamiento. Madrid: Visión Libros.

Durango Vanegas, C. E. (2015). Asociación de datos espacio-temporales en bases de datos Oracle. Ingenierías USBmed, 5(2), 100-108.

Febles Díaz, O., Febles Rodríguez, J. P., Estrada Sentí, V., \& Díaz Márquez, I. (2012). SI-Holmes: aplicación compuesta para la gestión de sistemas basados en conocimiento. Acimed,, 23(3), 295-304.

Fernández, M., \& Gómez Pérez, A. (2002). Overview and analysis of methodologies for building ontologies. The Knowledge Engineering Review, 17(2), 129-156.

Fernández, R. (2011). Informatización de la Gestión de las Redes Eléctricas. (Tesis en opción al título de Doctor en Ciencias Técnicas), Universidad Central "Marta Abreu" de Las Villas, Santa Clara.

Garea Llano, E. (2007). Estado actual de la interpretación semántica de datos espaciales. Blue $\begin{array}{lllll}\text { Series. } & \text { Pattern } & \text { Recognition. }\end{array}$ http://www.cenatav.co.cu/doc/RTecnicos/RT\%20SerieAzul 001web.pdf

González, C., Pérez, R., Vásquez Stanescu, C., \& Araujo, G. (2014). Eficiencia Energética: Uso racional de la energía eléctrica en el sector administrativo: Ministerio del Poder Popular para la Energía Eléctrica.

González Castellanos, M. (2010). Extensión de algoritmos representativos del aprendizaje automático al trabajo con datos tipo conjunto. (máster), Universidad Central "Marta Abreu" de Las Villas, Santa Clara, Villa Clara. Retrieved from http://dspace.uclv.edu.cu:8089/handle/123456789/5698

Gotoh, O. (1982). An Improved Algorithm for Matching Biological Sequences. Journal of Molecular Biology, 162(3), 705-708.

Gruber, T. R. (1993). A translation approach to portable ontologies (Vol. 5). Padova, Italy:: Knowledge acquisitions.

Guzmán, Y. M. a. M. V. (2009). Modelo de evaluación para software que emplean indicadores métricos en la vigilancia científico-tecnológica.

Ibarra Ruiz, G. C. (2013). Aplicaciones del sistema de información georeferenciado en el Ecuador. Revista Científica Yachana, 2(2), 279-282.

Jaro, M. A. (1976). Unimatch: A Record Linkage System: User's Manual. US Bureau of the Census.

Larin Fonseca, R., \& Garea Llano, E. (2009). Integración semántica de datos espaciales con sistemas de información geográfica. In S. Azul (Ed.), Reconocimiento de Patrones (Vol. 2142). Ciudad de La Habana,Cuba: CENATAV. 
Larin Fonseca, R., \& Garea Llano, E. (2013). Enfoque de integración semántica de datosgeoespaciales a bajo nivel de abstracción. Paper presented at the Informática 2013, La Habana, Cuba.

Machado García, N., González Ruiz, L., \& Balmaseda Espinosa, C. (2014). Recuperación de objetos geoespaciales utilizando medidas de similitud semántica. Revista Cubana de Ciencias Informáticas, 8(2), 132 - 143.

Morell Pérez, C. A. (2005). Extensiones al razonamiento basado en casos para su aplicación en la planificación de procesos. (Doctoral), Universidad Central "Marta Abreu" de Las Villas, Santa Clara, Villa Clara.

Oliva Santos, R., Garea Llano, E., \& Macia Perez, F. (2009). Ideas para una arquitectura de persistencia basada en geoontologías para anotaciones semánticas de datos geográficos. Desarrollo de Grandes Aplicaciones de Red. Paper presented at the JDARE 2009.

Ramírez Bustamante, F., \& López Díaz, E. (2006). Spelling Error Patterns in Spanish for Word Processing Applications Paper presented at the Fifth international conference on Language Resources and Evaluation, LREC 2006, Cotone Conference Center, GENOA - ITALY

Rivero Hernández, D. d. I. C., Vila Labrada, J., \& Rivero Hernández, M. (2014). Ontologías. Integración De Esquemas. Tlatemoani, 17.

Short, T. A. (2004). Electric Power Distribution Handbook. Boca Ratón, Florida.

Smith, T. F., \& Waterman, M. S. (1981). Identification of common molecular subsequences. Journal of Molecular Biology, 147(1), 195-197.

Tolaba, A. C., Caliusco, M. L., \& Galli, M. R. (2014). Representación del Conocimiento de la Información Geográfica siguiendo un Enfoque basado en Ontologías. RISTI - Revista Ibérica de Sistemas e Tecnologias de Informação, 14, 101-116.

Vera, F., \& Garea Llano, E. (2009). Alineamiento de ontologías en el dominio geoespacial. In S. Azul (Ed.), Reconocimiento de Patrones (Vol. 2142, pp. 74). Ciudad de La Habana,Cuba: CENATAV.

Winkler, W. E. (2000). "Using the EM Algorithm for Weight Computation in the Fellegi-Sunter Model of Record Linkage". Proceedings of the Section on Survey Research Methods, 667671. 